\title{
Commentary: Synergistic tumoricidal effect of combined hPD-L1 vac- cine and HER2 gene vaccine
}

\author{
Wangqian Zhang', Cun Zhang ${ }^{2 *}$
}

${ }^{1,2}$ State Key Laboratory of Cancer Biology, Biotechnology Center, School of Pharmacy, Fourth Military Medical University, Xi'an, China, 710032

\section{Article Info}

\section{Article Notes}

Received: May 03, 2018

Accepted: May 29, 2018

*Correspondence:

Dr. Cun Zhang

State Key Laboratory of Cancer Biology, Biotechnology

Center, School of Pharmacy, Fourth;

Military Medical University, X'an, China

Telephone: +86 (0) 29-84773490

Fax: +86 (0) 2984774773

Email: zhangcun@fmmu.edu.cn

(c) 2018 Zhang C. This article is distributed under the terms of the Creative Commons Attribution 4.0 International License.

\section{Introduction}

Breast cancer is the most frequent tumor worldwide and 252,710 women have developed the disease in the United States in 20171. Breast cancer is a heterogeneous disease and is clinically classified based on presence of hormonal receptors for estrogen and progesterone, and the expression/amplification status of the HER2 (human epidermal growth factor receptor 2)-protein/oncogene ${ }^{[2]}$. HER2 is a tumor associated antigen which is over-expressed in a subset of patients with breast cancer $(20-30 \%)^{2}$. There is abundant evidence that HER2 is a predictive target of clinical benefit to passive immune therapy with HER2-directed monoclonal antibodies (i.e., trastuzumab, pertuzumab, and TDM-1) alone or in combination with chemotherapy for the treatment of HER2 positive breast cancer-5.

Since HER2 is selectively overexpressed in cancer cells, and known to be effectively suppressed by antibodies targeting extracellular and intracellular domains, HER2 is also a good candidate for cancer vaccines ${ }^{6}$. However, in the process of practical study of tumor vaccine, it suffered many kinds of setbacks that the immune systems do not cause tumor regression.

In our recent study? , "Synergistic tumoricidal effect of combined hPD-L1 vaccine and HER2 gene vaccine", an attempt has been made to demonstrate that PD-L1/HER2 gene vaccine combination therapy synergistically generates marked tumoricidal effects against established HER2-expressing cancers and it provides new idea and evidence for designing tumor vaccine which not only can induce strong tumor-specific immune response, but also can block tumor immune escape.

\section{HER2 directed vaccines}

Several HER2-directed vaccines are currently undergoing clinical development in hopes to trigger robust Th1 response against HER2positive breast cancer. For example, E75 (aa369-377) vaccine is a nine-amino acid peptide which elicits E75 immune response in activating $\mathrm{CD}^{+}$and $\mathrm{CD}^{+}{ }^{+} \mathrm{Th} 1$ responses against HER2. One limitation of E75 vaccines is restriction to HLA-A2 and -A3 subtypes ${ }^{8}$. Similarly, the GP2 (aa654-662) vaccine from the trans-membrane domain of HER2 also binds to HLA class I with low affinity9. Responses to GP2 are usually short-lived likely because the lack of robust activation of other components of the immune system, such as $\mathrm{CD} 4^{+} \mathrm{T}$-helper cells ${ }^{10}$. Furthermore, the over-expression of HER 2 by breast cancer cells causing reduced levels of MHC class I, and decreased number of molecules of 
the antigen processing and presenting machinery (APM), thus resulting in a tumor escape from immune-surveillance ${ }^{11}$. However, in the process of practical study of tumor vaccine, it suffered many kinds of setbacks that the vaccine immunity effect independent employment effect is not good.

\section{Tumor Immune Escape and Immune Checkpoints}

As we know, there are many kinds of mechanism for tumor immune escape ${ }^{12-14}$. For example, the lack of MHC I molecules on the tumor cell surface led to the activation barrier of tumor-specific CTL. Similarly, the lack expression of co-stimulatory molecules B7 family, some cytokines (TGF $\beta$ ) secreted by tumor cells or soluble cytokine receptor analogs all inhibited an effective anti-tumor immune response. In recent years, more and more studies aimed at the effects of the negative immune checkpoints on tumor immune escape. The negative immune checkpoints included CTLA-4? PD-1, the B7 family molecule (PD-L1, PD$\mathrm{L} 2)$, and $\mathrm{CD} 4^{+} \mathrm{CD} 25^{+}$Tregs. Their physiological function is to regulate the strength and breadth of the immune response, thus avoiding the damage of normal tissue. Tumor cells are capable of high expressing or inducing the production of immune system "negative regulation point" to escape the attack of immune system. There is growing evidence that blocking one or more "negative regulation point" can produce a lasting and effective anti-tumor immune response. For example, the antibody against CTLA-4, ipilimumab, has been approved by the US Food and Drug Administration (FDA) to treat metastatic melanoma. The antibody against programmed death-1(PD-1), nivolumab and pembrolizumab have been approved by FDA to treat non-small-cell lung cancer.

\section{PD-L1 directed vaccines}

PD-L1, the homolog of B7.1/2 (CD80/86), shows the ability of co-inhibitory molecules and regulates the immune system 15,16 . The PD-L1 protein is a cell surface glycoprotein which is only expressed on macrophage lineage of cells in normal tissues. Under physiological conditions, the PD-1/ PD-L1 signaling pathway inhibits transmission of signals from the activated $\mathrm{T}$ cell receptor, plays a key role in selftolerance ${ }^{17} \underline{18}$ and prevents $\mathrm{T}$ cells from over-activation and tissue damage during infection $\frac{19}{}$.The expression of PD-L1 is elevated in many types of cancer and is often correlated with poor patient prognosis. Ligation of PDL1 on cancer cells with PD-1 on tumor-specific $\mathrm{T}$ cells has been demonstrated to suppress T-cell activation and proliferation, and to induce T-cell apoptosis. Tumor cells exploit this regulatory interaction as a mechanism of immune evasion. More recently, the antibody against PD-L1 (MPDL3280A) has been approved by FDA for the treatment of PD-L1-positive NSCLC $\frac{20}{}$. Our previous study also showed that the TT-rhPD-L1IgV vaccine induced hightiter anti-PD-L1 antibody, which could inhibit the growth of PD-L1-positive SP20 metastatic tumor ${ }^{21}$. We thought that PD-L1 vaccine could not only kill the PD-L1-positive tumor cells like other common tumor-associated antigen vaccines, more importantly, it could block tumor immune escape and increase the body's anti-tumor immune response by blocking $\mathrm{PD}-1 / \mathrm{PD}-\mathrm{L} 1$ pathway whether the tumors expressed PD-LI or not.

\section{Combination Therapy of hPD-L1 vaccine and HER2 gene vaccine}

As previously mentioned, PD-L1 act as a tumor associated antigen and an immune-suppressor playing an important role in the immune escape of tumor cells. Our studies suggest that in tumor-bearing mice, EMT6 may secrete IFN- $\gamma$ and promote self-expression of PD-L1 to evade the immune system attacks. Then, the positive feedback loops of tumor cells immune escape were formed and promote their own growth. The antibody induced by PD-L1 vaccine could not directly kill tumor cells, but combined with the PD-L1 located on the surface of tumor cells, to form a molecular barrier, blocked the mediated immune suppression and improved the reactivity of immune cell. As a DNA vaccine, HER2 gene vaccine not only possesses the ability to induce both $\mathrm{T}$ cell and humoral immune responses. After the combination therapy of hPD-L1 vaccine and HER2 gene vaccine, the rhPD-L1 protein vaccine increased the HER2specific humoral and cellular immunity responses against EMT6 tumors, and thus, promoted the HER2 gene vaccine to induce a better anti-tumor immune response. So, the study suggests that PD-L1 vaccine might also enhance the efficacy of other tumor-associated antigen vaccine, antibody and immunotherapy approaches. However, the two newly published papers have demonstrated that in some tumors, the sequence and timing of antibody treatment targeting both costimulatory and inhibitory receptors is critical to success of the combined therapy, and sequential treatment has the combined benefit of both optimizing the antitumor immune response as well as potentially minimizing possible toxicity from acute cytokine release ${ }^{22,23}$. Therefore, the role of the PD-L1 vaccine in combination with other immunotherapies in cancer patients and the sequence and timing of the PD-L1 vaccine treatment remain to be further studied.

\section{Conflict of interest}

The authors declare that there are no conflicts of interest.

\section{Acknowledgements}

The authors thank the members of the Biotechnology Center of the Fourth Military Medical University for their excellent technical support. This work was supported by the National Natural Foundation of China, NSFC81472484, 81472649, 81672864 . 


\section{References}

1. Siegel RL, Miller KD, Jemal A. Cancer Statistics, 2017. CA Cancer J Clin. 2017; 67(1): p. 7-30.

2. Costa RLB, Soliman H, Czerniecki BJ. The clinical development of vaccines for HER2(+) breast cancer: Current landscape and future perspectives. Cancer Treat Rev. 2017; 61: p. 107-115.

3. Baselga J, Cortés J, Kim SB, et al. Pertuzumab plus trastuzumab plus docetaxel for metastatic breast cancer. N Engl J Med. 2012; 366(2): p. 109-19.

4. Cobleigh MA, Vogel CL, Tripathy D, et al. Multinational study of the efficacy and safety of humanized anti-HER2 monoclonal antibody in women who have HER2-overexpressing metastatic breast cancer that has progressed after chemotherapy for metastatic disease. J Clin Oncol. 1999; 17(9): p. 2639-48.

5. Salgado R, Denkert C, Campbell C, et al. Tumor-Infiltrating Lymphocytes and Associations With Pathological Complete Response and Event-Free Survival in HER2-Positive Early-Stage Breast Cancer Treated With Lapatinib and Trastuzumab: A Secondary Analysis of the NeoALTTO Trial. JAMA Oncol. 2015; 1(4): p. 448-54.

6. Renard V, Leach DR. Perspectives on the development of a therapeutic HER-2 cancer vaccine. Vaccine. 2007; 25 Suppl 2: p. B17-23.

7. Zhang W, Wang S, Gu J, et al. Synergistic tumoricidal effect of combined hPD-L1 vaccine and HER2 gene vaccine. Biochem Biophys Res Commun. 2018; 497(1): p. 394-400.

8. Patil R, Clifton GT, Holmes JP, et al. Clinical and immunologic responses of HLA-A3+ breast cancer patients vaccinated with the HER2/neuderived peptide vaccine, E75, in a phase I/II clinical trial. J Am Coll Surg. 2010; 210(2): p. 140-7.

9. Mittendorf EA, Storrer CE, Foley RJ, et al. Evaluation of the HER2/neuderived peptide GP2 for use in a peptide-based breast cancer vaccine trial. Cancer. 2006; 106(11): p. 2309-17.

10. Knutson KL, Schiffman $\mathrm{K}$, Cheever MA, et al. Immunization of cancer patients with a HER-2/neu, HLA-A2 peptide, p369-377, results in short-lived peptide-specific immunity. Clin Cancer Res. 2002; 8(5): p. 1014-8.

11. Mimura $\mathrm{K}$, Ando $\mathrm{T}$, Poschke $\mathrm{I}$, et al. $\mathrm{T}$ cell recognition of HLA-A2 restricted tumor antigens is impaired by the oncogene HER2. Int Cancer. 2011; 128(2): p. 390-401.

12. Korman AJ, Peggs KS, Allison JP. Checkpoint blockade in cancer immunotherapy. Adv Immunol. 2006; 90: p. 297-339.

13. Waldmann T.A. Effective cancer therapy through immunomodulation. Annu Rev Med. 2006; 57: p. 65-81.

14. Bowtell DD, Drapkin R. Highlights from the 2014 American Association for Cancer Research Annual Meeting. Gynecol Oncol. 2014; 134(1): p. 3-5.

15. Butte MJ, Keir ME, Phamduy TB, et al. Programmed death-1 ligand 1 interacts specifically with the B7-1 costimulatory molecule to inhibit T cell responses. Immunity. 2007; 27(1): p. 111-22.

16. Horne-Debets JM, Faleiro R, Karunarathne DS, et al. PD-1 dependent exhaustion of CD8+ T cells drives chronic malaria. Cell Rep. 2013; 5(5): p. 1204-13.

17. Keir ME, Latchman YE, Freeman GJ, et al. Programmed death-1 (PD1):PD-ligand 1 interactions inhibit TCR-mediated positive selection of thymocytes. J Immunol. 2005; 175(11): p. 7372-9.

18. Liang SC, Latchman YE, Buhlmann JE, et al. Regulation of PD-1, PD-L1, and PD-L2 expression during normal and autoimmune responses. Eur J Immunol. 2003; 33(10): p. 2706-16.

19. Isogawa M, Furuichi Y, Chisari FV. Oscillating CD8(+) T cell effector functions after antigen recognition in the liver. Immunity. 2005; 23(1): p. 53-63.

20. Jelinek T, Hajek R. PD-1/PD-L1 inhibitors in multiple myeloma: The present and the future. Oncoimmunology. 2016; 5(12): p. e1254856.

21. Zhang C, Wang W, Qin X, et al. B7-H1 protein vaccine induces protective and therapeutic antitumor responses in SP2/0 myelomabearing mice. Oncol Rep. 2013; 30(5): p. 2442-8.

22. Shrimali RK, Ahmad S, Verma V, et al. Concurrent PD-1 Blockade Negates the Effects of OX40 Agonist Antibody in Combination Immunotherapy through Inducing T-cell Apoptosis. Cancer Immunol Res. 2017; 5(9): p. 755-766.

23. Messenheimer DJ, Jensen SM, Afentoulis ME, et al. Timing of PD-1 Blockade Is Critical to Effective Combination Immunotherapy with Anti-OX40. Clin Cancer Res. 2017; 23(20): p. 6165-6177. 